\title{
Construction of Interval-valued Fuzzy Preference Relations using Ignorance Functions. Interval-valued non dominance Criterion
}

\author{
E. Barrenechea, A. Fernández, F. Herrera, H. Bustince
}

\begin{abstract}
In this work we present a construction method for interval-valued fuzzy preference relations from a fuzzy preference relation and the representation of the lack of knowledge or ignorance that experts suffer when they define the membership values of the elements of that fuzzy preference relation. We also prove that, with this construction method, we obtain membership intervals for an element which length is equal to the ignorance associated with that element. We then propose a generalization of Orlovsky's non dominance method to solve multi-criteria decision making problems using interval-valued fuzzy preference relations.
\end{abstract}

\section{Introduction}

We know that there exist problems for which the solution obtained by means of fuzzy techniques sometimes are very good, but some others not. Usually, this discordance is due to the choice by the experts of the membership functions to represent the information. For instance, in decision making problems experts express their preferences with a numerical value, depending on the knowledge they have about them. Sometimes, experts suffer from a great lack of knowledge about the environment where the fuzzy decision making method is going to be applied. In these cases, the numerical values they provide are not the more suitable ones to represent the preferences, and hence the result is not the best one (see [5, 14]).

Once the fuzzy preference relation $(F P R)$ for a decision making problem is known, the goal is to improve the solution that is obtained with common fuzzy

Edurne Barrenechea, Humberto Bustince

Dept. Automática y Computación, Universidad Púbilica de Navarra, e-mail: \{edurne.barrenechea,bustince\}@unavarra.es

Alberto Fernández, Francisco Herrera

Dept. of Computer Science and Artificial Intelligence, Universidad de Granada e-mail: \{alberto,herrera\}@decsai.ugr.es 
methods, as Orlovsky's non dominance method (see [7]), using interval-valued fuzzy sets (see $[12,18]$ ).

To achieve this goal we will measure the ignorance (lack of knowledge) of the expert when providing the membership values of the elements of the FPR. We will do this using ignorance functions (see [2]). So each element will be associated with two values: the first value is given by the expert, and corresponds to the degree of membership of the element to the original $F P R$; the second value is calculated with the ignorance function and represents the lack of knowledge of the expert in the assignation of the first value. From these two values we will build an interval-valued fuzzy preference relation $(I V F P R)$.

For the new IVFPR we introduce a generalization of the non dominance method, which allows to recover the classical algorithm solution.

This work is organized as follows: In the next section we introduce the basic necessary concepts. In Section 3 we introduce the relationship between the concept of strict fuzzy preference relation given by Fodor and Roubens and the one given by Orlovsky. In Section 4 we consider a construction method of interval-valued fuzzy preference relations from fuzzy preference relations and ignorance functions. In Section 5, we propose a generalization of the non-dominance criterion proposed by Orlovsky to solve decision making problems. We finish with some conclusions and future lines of research.

\section{Preliminary definitions}

In fuzzy set theory, we know that a function $\mathbf{N}:[0,1] \rightarrow[0,1]$, with $\mathbf{N}(0)=1, \mathbf{N}(1)=$ 0 that is strictly decreasing and continuous, is called strict negation. If $\mathbf{N}$ is also involutive, then it is a strong negation.

Definition 1. [17] A fuzzy set $\tilde{A}$ on a finite universe $U$ is a mapping $U \rightarrow[0,1]$.

We will denote by $F S(U)$ the set of all the fuzzy sets on $U$.

Let us denote by $L([0,1])$ the set of all closed subintervals in $[0,1]$, that is,

$$
L([0,1])=\left\{\mathbf{x}=[\underline{x}, \bar{x}] \mid(\underline{x}, \bar{x}) \in[0,1]^{2} \text { and } \underline{x} \leq \bar{x}\right\} .
$$

We also denote $0_{L}=[0,0], 1_{L}=[1,1]$ and the length of $\mathbf{x} \in L([0,1])$ as $W(\mathbf{x})=\bar{x}-\underline{x}$.

Definition 2. [18] An interval-valued fuzzy set $A$ on a universe $U$ is a mapping $A: U \rightarrow L([0,1])$.

Note that the membership of each element $u_{i} \in U$ is given by $A\left(u_{i}\right)=\left[\underline{A}\left(u_{i}\right), \bar{A}\left(u_{i}\right)\right]$. We will denote by $I V F S(U)$ the set of all interval-valued fuzzy sets on $U$.

An IV negation is a function $N_{I V}: L([0,1]) \rightarrow L([0,1])$ that is decreasing (with respect to the order: $\mathbf{x} \leq_{L} \mathbf{y}$ if and only $\underline{x} \leq y$ and $\left.\bar{x} \leq \bar{y}\right)$ and with $N_{I V}\left(1_{L}\right)=0_{L}$ and $N_{I V}\left(0_{L}\right)=1_{L}$. If for all $\mathbf{x} \in L([0,1]), N_{I V}\left(N_{I V}(\mathbf{x})\right)=\mathbf{x}, N_{I V}$ is said to be involutive. 
Theorem 1. [3] A function $N_{I V}: L([0,1]) \rightarrow L([0,1])$ is an involutive IV negation if and only if there exists an involutive negation $\mathbf{N}$ such that

$$
N_{I V}(\mathbf{x})=[\mathbf{N}(\bar{x}), \mathbf{N}(\underline{x})] .
$$

Throughout this paper we use involutive IV negations $N_{I V}$ generated from the standard negation $\mathbf{N}(x)=1-x$ for all $x \in[0,1]$ in such a way that $N_{I V}(\mathbf{x})=$ $[\mathbf{N}(\bar{x}), \mathbf{N}(\underline{x})]=[1-\bar{x}, 1-\underline{x}]$.

A triangular norm (t-norm for short) $T:[0,1]^{2} \rightarrow[0,1]$ is an associative, commutative, non-decreasing function such that $T(1, x)=x$ for all $x \in[0,1]$. A t-norm $T$ is called idempotent if, $T(x, x)=x$ for all $x \in[0,1]$.

Three basic t-norms are the following: the minimum $T_{M}(x, y)=\min (x, y)$, the product $T_{P}(x, y)=x \cdot y$ and the Eukasiewicz $T_{\mathrm{E}}(x, y)=\max (x+y-1,0)$.

In this paper we will also use the following relationship on $L([0,1])$ (see [15]): let $\mathbf{x}, \mathbf{y} \in L([0,1])$ and let $s(\mathbf{x})=\underline{x}+\bar{x}-1$ and $s(\mathbf{y})=y+\bar{y}-1$ be the scores of $\mathbf{x}$ and $\mathbf{y}$ respectively. Let $h(\mathbf{x})=1-(\bar{x}-\underline{x})$ and $h(\mathbf{y})=\overline{1}-(\bar{y}-\underline{y})$ be the accuracy degrees of $\mathbf{x}$ and $\mathbf{y}$ respectively. Then

1. If $s(\mathbf{x})<s(\mathbf{y})$, then $\mathbf{x}<\mathbf{y}$;

2. If $s(\mathbf{x})=s(\mathbf{y})$, then

2.1 If $h(\mathbf{x})=h(\mathbf{y})$, then $\mathbf{x}=\mathbf{y}$;

2.2 if $h(\mathbf{x})<h(\mathbf{y})$, then $\mathbf{x}<\mathbf{y}$.

The relation between the score function $s$ and the accuracy function $h$ is similar to the relation between the mean and the variance in statistics. Observe that any two intervals are comparable with this order relation. Moreover, it follows easily that $0_{L}$ is the smallest element in $L([0,1])$ and $1_{L}$ is the largest.

\section{Fuzzy binary preference relations and interval-valued fuzzy binary preference relations}

First, we recall the concept of strict fuzzy binary preference relation given by Fodor and Roubens [4] and relate it with the definition given, for the same concept, by Orlovsky in [7]. Later, we recall the definition of interval-valued fuzzy binary preference relations and the reciprocity property.

\subsection{Strict Fuzzy binary preference relations}

Let $R \in F R(X \times X)$ be a fuzzy preference relation over a set of alternatives $X=$ $\left\{x_{1}, \ldots, x_{n}\right\}$ (see $[1,4,5,8]$ ); for each pair of alternatives $x_{i}$ and $x_{j}, R_{i j}=R\left(x_{i}, x_{j}\right)$ 
represents a degree of (weak) preference of $x_{i}$ over $x_{j}$, namely the degree to which $x_{i}$ is considered as least as good as $x_{j}$.

From a weak preference relation $R$, Fodor and Roubens [4] (see also [1, 11]) derive the following relation:

A Strict preference $P_{i j}=P\left(x_{i}, x_{j}\right)$ is a measure of strict preference of $x_{i}$ over $x_{j}$, indicating that $x_{i}$ is (weakly) preferred to $x_{j}$ but $x_{j}$ is not (weakly) preferred to $x_{i}$.

More specifically, Fodor and Roubens propose to express the above relation in terms of a t-norm $T$ and a strict negation $\mathbf{N}$ :

$$
P_{i j}=T\left(R_{i j}, \mathbf{N}\left(R_{j i}\right)\right) \text { for all } i, j \in\{1, \ldots, n\} ;
$$

Fuzzy preference structures have been studied deeply as their axiomatic construction (see $[4,1,7,9,10])$.

A fuzzy preference relation $R$ satisfies the property of reciprocity if $R_{i j}+R_{j i}=1$ for all $i, j \in\{1, \cdots, n\}$. In reciprocal preference relations is usual not to define the elements in the diagonal (see [6]).

Proposition 1. Let $R$ be a reciprocal fuzzy preference relation and $\mathbf{N}(x)=1-x$ for all $x \in[0,1]$. Then,

$$
P_{i j}=R_{i j} \text { if and only if } T=T_{M}
$$

for all $R_{i j} \in R$.

Orlovsky in [7] gives the following definition of strict fuzzy preference relation $R \in F R(X \times X)$ :

$$
R_{i j}^{s}=\left\{\begin{array}{ll}
R_{i j}-R_{j i} & \text { if } R_{i j}>R_{j i} \\
0 & \text { otherwise }
\end{array} .\right.
$$

Next, we present the relationship between strict fuzzy preference relation given by Fodor and Roubens ([4]) $P_{i j}$ and the one given by given by Orlovsky [7] $R_{i j}^{s}$.

Lemma 1. If $T=T_{€}$ and $\mathbf{N}(x)=1-x$ for all $x \in[0,1]$, then

$$
P_{i j}=R_{i j}^{s}, \quad \text { for all } i, j \in\{1, \ldots, n\} \text {. }
$$

\subsection{Interval-valued reciprocal preference relations}

A first approach to add some flexibility to the uncertainty representation problem is by means of interval-valued fuzzy relations. An Interval-valued fuzzy binary relation $\mathbf{r}$ on $X$ is defined as an interval-valued fuzzy subset of $X \times X$; that is, $\mathbf{r}: X \times X \rightarrow L([0,1])$. The interval $\mathbf{r}\left(x_{i}, x_{j}\right)=\mathbf{r}_{i j}$ denotes the degree to which elements $x_{i}$ and $x_{j}$ are related in the relation $\mathbf{r}$ for all $x_{i}, x_{j} \in X$. By $\operatorname{IVFR}(X \times X)$ we denote the set of all interval-valued fuzzy relations on $X \times X$.

Definition 3. Let $\mathbf{r} \in \operatorname{IVFR}(X \times X)$. We say that $\mathbf{r}$ satisfies the reciprocity property if for all $\mathbf{r}_{i j}, \mathbf{r}_{j i} \in \mathbf{r}$ the following identities hold: 


$$
\begin{aligned}
& \underline{r}_{i j}+\bar{r}_{j i}=1 \\
& \underline{r}_{j i}+\bar{r}_{i j}=1
\end{aligned}
$$

In this work we use interval-valued fuzzy preference relations that satisfy the reciprocity property and such that the elements in their main diagonal are not defined.

\section{Construction of interval-valued fuzzy preference relations from fuzzy preference relations and weak ignorance functions}

The goal of this section is to build interval-valued fuzzy preference relations arising from a fuzzy preference relation. For this purpose, we use the concept of weak ignorance function and a new construction method of intervals.

\subsection{Weak ignorance}

The concept of ignorance functions is defined in [2] in order to quantify the lack of knowledge of an expert when he or she assigns a numerical value to the membership of an object to a given class and another numerical value for the membership of the same element to a different class.

Definition 4. [2] An ignorance function is a continuous mapping $G_{i}:[0,1]^{2} \rightarrow[0,1]$ such that:

$\left(G_{i} 1\right) \quad G_{i}(x, y)=G_{i}(y, x)$ for all $x, y \in[0,1]$;

$\left(G_{i} 2\right) \quad G_{i}(x, y)=0$ if and only if $x=1$ or $y=1$;

$\left(G_{i} 3\right)$ If $x=0.5$ and $y=0.5$, then $G_{i}(x, y)=1$;

$\left(G_{i} 4\right) \quad G_{i}$ is decreasing in $[0.5,1]^{2}$;

$\left(G_{i} 5\right) \quad G_{i}$ is increasing in $[0,0.5]^{2}$.

Observe that this definition implies that we have assumed that a value of 0.5 corresponds to complete lack of knowledge of the expert on the membership of an element to a class.

In order to build the interval-valued fuzzy sets the authors define in [13] a new function called weak ignorance for modeling the uncertainty associated with the definition of the membership functions. From this new concept, they represent the linguistic labels using by means of interval-valued fuzzy sets and present a natural extension of both the Fuzzy Reasoning Method (FRM) and the computation of the rule weight.

Proposition 2. [13] Let $G_{i}:[0,1]^{2} \rightarrow[0,1]$ be an ignorance function. The mapping: 


$$
\begin{aligned}
& g:[0,1] \rightarrow[0,1] \text { given by } \\
& g(x)=G_{i}(x, 1-x)
\end{aligned}
$$

is a continuous function that satisfies:

(i) $g(x)=g(1-x)$ for all $x \in[0,1]$;

(ii) $g(x)=0$ if and only if $x=0$ or $x=1$;

(iii) $g(0.5)=1$.

Definition 5. [13]A continuous mapping $g:[0,1] \rightarrow[0,1]$ is called weak ignorance function if it satisfies the items $(i)-($ iii) in Proposition 2.

The name is due to the fact that they are only associated with an element, in the sense that they depend on a single variable, and not of two. We understand weak ignorance functions as a quantification of the lack of knowledge an expert suffers from when assigning a numerical value to the membership of an object to a given class (set).

Example 1. The function $g(x)=2 \cdot \min (x, 1-x)$ for all $x \in[0,1]$, is a weak ignorance function.

\subsection{Construction of interval-valued fuzzy preference relations}

One of the main goals of this work is to build an $I V F R$ arising from a $F R$, in such a way that for each element, the length of the interval that represents the membership to the new relation, is equal to the weak ignorance associated with the membership degree of the same element to the original fuzzy relation.

Proposition 3. Let $R \in F R(X \times X)$. In the setting of Proposition 2 the following items hold:

1. The relation $\mathbf{r}$ given by

$$
\mathbf{r}_{i j}= \begin{cases}{\left[\left(R_{i j}^{s} \cdot\left(1-g\left(R_{i j}\right)\right), R_{i j}^{s} \cdot\left(1-g\left(R_{i j}\right)\right)+g\left(R_{i j}\right)\right]\right.} & \text { if } R_{i j}>R_{j i} \\ {\left[0, g\left(R_{i j}\right)\right]} & \text { otherwise }\end{cases}
$$

for all $i, j$ is an interval-valued fuzzy relation on $X \times X$;

2. $W\left(\mathbf{r}_{i j}\right)=g\left(R_{i j}\right)$ for all $R_{i j} \in R$;

3. If $R$ satisfies the reciprocity property, then the interval-valued fuzzy preference relation $\mathbf{r}$ given by item 1 . also satisfies it (in the sense of Eq. (3)); 


\section{An approach to multi-criteria decision making with interval-valued fuzzy preference relations}

In this section we propose a generalization of the non-dominance criterion proposed by Orlovsky. We always consider normalized fuzzy preference relations to satisfy the reciprocity property. In the algorithm we will use the construction method given in Proposition 4 (item 1.) and the concept of weak ignorance function.

Given a fuzzy preference relation $R^{*} \in F R(X \times X)$, to normalize such relation to $[0,1]$ we use Eq. (6), in such a way that for each element of the new relation it holds that $R_{i j}=1-R_{j i}$.

$$
R_{i j}=\left\{\begin{array}{l}
\frac{R_{i j}^{*}}{R_{i j}^{*}+R_{j i}^{*}} \text { if } R_{i j}^{*}+R_{j i}^{*} \neq 0 \\
0 \text { othercase }
\end{array}\right.
$$

From the normalized fuzzy preference relation $R$ we must extract a set of nondominated alternatives as the solution of the decision making problem. Specifically, the maximal non-dominated elements of $R$ are calculated by means of the following operations, according to the non-dominance criterion proposed by Orlovsky in [7]:

Step 1. Compute the fuzzy strict preference relation $R^{s}$ as indicated in Eq. (2);

Step 2. Compute the non-dominance degree of each alternative $N D_{i}$ in the following way:

$$
N D_{i}=1-\max _{j}\left\{R_{j i}^{s}\right\}
$$

This value represents the degree to which the alternative $i$ is dominated by one of the remaining alternatives.

Step 3. Take as the best alternative that corresponding to the index of the maximal nondominance value:

$$
\text { Alternative }\left(x_{p}\right)=\arg \max _{i=1, \cdots, n}\left\{N D_{i}\right\}
$$

We must point out that it could happen that there exist two or more alternatives with the same degrees of membership to the set ND. In this case, the algorithm does not choose any of those alternatives. This fact has led many authors to propose other algorithms (see [5, 14, 16]).

The main idea of our approach is to build an interval-valued fuzzy preference relation from the strict preference relation $R^{s}$ (Step 1) of the non-dominance algorithm.

Given a fuzzy preference relation $R^{*}$ (without defined elements in the main diagonal) and given a weak fuzzy ignorance function $g$ in the sense of Proposition 2 , the algorithm that we propose is the following: 
(NDIVA1) Construct $R$ normalizing the fuzzy preference relation $R^{*} \in F R(X \times X)$ by means of Eq. (6);

(NDIVA2) Compute the fuzzy strict preference relation $R^{s}$ using Eq. (2);

(NDIVA3) Build the interval-valued fuzzy relation $\mathbf{r}$ using Eq. (5):

$$
\mathbf{r}_{i j}= \begin{cases}{\left[\left(R_{i j}^{s} \cdot\left(1-g\left(R_{i j}\right)\right), R_{i j}^{s} \cdot\left(1-g\left(R_{i j}\right)\right)+g\left(R_{i j}\right)\right]\right.} & \text { if } R_{i j}>R_{j i} \\ {\left[0, g\left(R_{i j}\right)\right]} & \text { otherwise }\end{cases}
$$

(NDIVA4) Build the interval-valued fuzzy set:

$$
\begin{aligned}
& N D_{I V}=\left\{\left(x_{j}, N D_{I V}\left(x_{j}\right)\right) \mid x_{j} \in X\right\} \text { where } \\
& N D_{I V}\left(x_{j}\right)=\mathbf{S}\left(\mathbf{r}_{i j}\right)=\left[\bigvee_{i=1}^{n}\left(\underline{r}_{i j}\right), \bigvee_{i=1}^{n}\left(\bar{r}_{i j}\right)\right]
\end{aligned}
$$

(NDIVA5) Apply the IV negation $N_{I V}$ generated by the standard negation to the set $N D_{I V}$; that is, build:

$$
N_{I V}\left(N D_{I V}\right)\left(x_{j}\right)=\left[1-\bigvee_{i=1}^{n}\left(\bar{r}_{i j}\right), 1-\bigvee_{i=1}^{n}\left(\underline{r}_{i j}\right)\right]
$$

(NDIVA6) Order the elements of the set $N_{I V}\left(N D_{I V}\right)$ in a decreasing way with respect to the interval order of the membership intervals.

(NDIVA7) If there exist several alternatives for which the intervals obtained in (NDIVA4) are such that the occupy the first place in the ordering of step (NDIVA5), take as solution alternative that with the biggest upper bound.

\section{Remark}

NDIVA Algorithm

- If for a majority of the elements $\mathbf{r}_{i j}$ given by Eq. (9) we have that $g\left(R_{i j}\right) \rightarrow 0$, i.e., if $R_{i j}^{s}=R_{i j}-R_{j i} \rightarrow 1$, then the resulting intervals have a very small length and it is reasonable to assume that the result obtained with the algorithm (NDIVA) is the same than the result obtained with the non-dominance algorithm.

This is due to the fact that if $R_{i j}-R_{j i} \rightarrow 1$, then $R_{i j}$ is very large and $R_{j i}$ is very small; that is, the expert is very sure about the preference of alternative $x_{i}$ against $x_{j}$. Moreover, in this case we have that the weak ignorance is very small. So, due to our construction method with Eq. (9) the intervals have a very small length.

- If for a majority of the elements $\mathbf{r}_{i j}$ given by Eq. (9) we have that $g\left(R_{i j}\right) \rightarrow 1$; i.e., if $R_{i j} \approx R_{j i} \approx 0.5$, then the (NDIVA) algorithm allows us to distinguish better than the non-dominance algorithm the alternative or alternatives that we must take as solution.

The reason for this is that if $R_{i j} \approx R_{j i} \approx 0.5$ and $R_{i j}+R_{j i}=1$, by Definition 4 we have that $g\left(R_{i j}\right)=g\left(R_{j i}\right) \rightarrow 1$, in such a way that the product $\left(R_{i j}-\right.$ 
$\left.R_{j i}\right)\left(1-g\left(R_{i j}\right)\right)$ goes to zero faster than $R_{i j}-R_{j i}$. So, in the (NDIVA) algorithm, for the cases for which the expert shows a great indifference for choosing one alternative or the other, we penalize (diminish) even more the difference of his or her preferences, ins such a way that, when we negate intervals in step (NDIVA5) we strengthen even more the worst possible cases, and the intervals obtained with Eq. (9) have a very large length.

Example 2. Let $X=\left\{x_{1}, x_{2}, x_{3}, x_{4}\right\}$ be the set of alternatives. Consider the normalized fuzzy relation

$$
R^{*}=\left(\begin{array}{cccc}
- & 0.70 & 0.65 & 0.30 \\
0.30 & - & 0.70 & 0.60 \\
0.35 & 0.30 & - & 0.70 \\
0.70 & 0.40 & 0.30 & -
\end{array}\right)
$$

For this algorithm we consider the weak ignorance function $g(x)=2 \cdot \min (x, 1-$ $x)$.

(NDIVA1) Construct $R$. In this case $R=R^{*}$.

(NDIVA2) Transform $R$ to $R^{s}$.

$$
R^{s}=\left(\begin{array}{cccc}
- & 0.40 & 0.30 & 0.00 \\
0.00 & - & 0.40 & 0.20 \\
0.00 & 0.00 & - & 0.40 \\
0.40 & 0.00 & 0.00 & -
\end{array}\right)
$$

(NDIVA3) Build the interval-valued fuzzy relation $\mathbf{r}$ :

$$
\mathbf{r}=\left(\begin{array}{cccc}
- & {[0.16,0.76]} & {[0.09,0.79]} & {[0.00,0.60]} \\
{[0.00,0.60]} & - & {[0.16,0.76]} & {[0.04,0.84]} \\
{[0.00,0.70]} & {[0.00,0.00]} & - & {[0.16,0.76]} \\
{[0.16,0.76]} & {[0.00,0.80]} & {[0.00,0.60]} & -
\end{array}\right)
$$

(NDIVA4) Build the interval-valued fuzzy set $N D_{I V}$ :

$N D_{I V}=\left\{\left(x_{1},[0.16,0.76]\right),\left(x_{2},[0.16,0.80]\right),\left(x_{3},[0.16,0.79]\right),\left(x_{4},[0.16,0.84]\right)\right\}$ (NDIVA5) Apply $N_{I V}$ to the interval-valued fuzzy sets $N D_{I V}$ :

$$
\begin{aligned}
& N_{I V}\left(N D_{I V}\right)= \\
& \left\{\left(x_{1},[0.24,0.84]\right),\left(x_{2},[0.20,0.84]\right),\left(x_{3},[0.21,0.84]\right),\left(x_{4},[0.16,0.84]\right)\right\}
\end{aligned}
$$

(NDIVA6) Order alternatives in a non-increasing way using the order relationship defined in terms of the score and accuracy functions.

$$
x_{1} \geq x_{3} \geq x_{2} \geq x_{4} .
$$




\subsection{Relationship between NDIVA algorithm and Orlovsky's algorithm}

Next, we present a new operator $P$ to associate a fuzzy set with each interval-valued fuzzy set satisfying a specific set of properties. If we apply this operator on the non dominance interval-valued fuzzy algorithm (Step NDIVA6) we recover the results given by Orlovsky's algorithm.

Definition 6. A $P$ operator is a mapping $L([0,1]) \rightarrow[0,1]$ given by:

$$
P(\mathbf{x})= \begin{cases}\frac{\underline{x}}{1-W(\mathbf{x})} & \text { if } W(\mathbf{x}) \neq 1 \\ 0 & \text { if } W(\mathbf{x})=1\end{cases}
$$

Proposition 4. Let $R \in F R(X \times X)$ and let $\mathbf{r} \in I V F R(X \times X)$ given by Eq. (5). In the setting of Proposition 2 the following items hold:

1. $P\left(\mathbf{r}_{i j}\right)=R_{i j}$ for all $R_{i j} \in R$ and for all $g\left(R_{i j}\right) \neq 1$.

2. If $g\left(R_{i j}\right)=1$ then, $P\left(\mathbf{r}_{i j}\right)=0$.

Proposition 5. Let P given in Eq. (15). The following properties hold:

1. $\underline{x} \leq P(\mathbf{x}) \leq \bar{x}$ for all $\mathbf{x} \in L([0,1])$;

2. $P([x, x])=x$ for all $x \in[0,1]$;

3. If $W(\mathbf{x}) \neq 1$, then $P(\mathbf{x})+P(1-\mathbf{x})=1$;

4. If $W(\mathbf{x})=1$, then $P(\mathbf{x})+P(1-\mathbf{x})=0$.

Remark Notice that in step (NDIVA5) of Example 2 all the elements have membership intervals with the same upper bound (see item (2) of Proposition 7). So, if for relation $R$ given by Eq. (12) we apply the non-dominance algorithm, all of the alternatives dominate with the same numerical value and we are not able of choosing the best one.

Proposition 6. Let $A_{I V} \in I V F S(U)$ and let $P$ the operator introduced in Definition 6. Then

$$
A=\left\{\left(u_{i}, P\left(A_{I V}\left(u_{i}\right)\right)\right) \mid u_{i} \in U\right\}
$$

is a fuzzy set on $U$,

Proposition 7. The following items hold:

1. Let $P$ be the operator given in Definition 6. If in the (NDIVA) algorithm we replace (NDIVA4) by:

(NDFS4) Build the set

$$
\begin{aligned}
& N D_{I V S}=\left\{\left(x_{j}, N D_{I V S}\left(x_{j}\right)\right) \mid x_{j} \in X\right\} \text { where } \\
& N D_{I V S}\left(x_{j}\right)=\left[\bigvee_{i=1}^{n} P\left(\mathbf{r}_{i j}\right), \bigvee_{i=1}^{n} P\left(\mathbf{r}_{i j}\right)\right]
\end{aligned}
$$


then we recover the non-dominance algorithm.

2. If in step (NDIVA6) we reorder the elements of the set $N_{I V}\left(N D_{I V}\right)$ in a nonincreasing way with respect to the upper bounds of the intervals, then the alternative(s) which are solution(s) for this algorithm are the same than those of the non-dominance algorithm.

Remark Notice that in step (NDIVA5) of Example 2 all the elements have membership intervals with the same upper bound (see item (2) of Proposition 7). So, if for relation $R$ given by Eq. (12) we apply the non-dominance algorithm, all of the alternatives dominate with the same numerical value and we are not able of choosing the best one.

\section{Conclusions}

In this paper we have presented an algorithm for decision making problems starting from a fuzzy preference relation. We use weak ignorance functions (in the sense of Proposition 2) to penalize indifference situations. That is, situations in which the preference of one alternative against the other is close to 0.5 . We also represent the preference degree of a relation by means of intervals such that their lengths is equal to the weak ignorance of the expert when he or she assigns a specific value.

Finally, we define a new operator that allows us to associate each interval-valued fuzzy set to a fuzzy sets. The analysis of this operator has allowed us to settle minimum conditions under which our first algorithm recovers the classical non dominance algorithm.

In the future we consider necessary to study a construction method to generalize the one presented in this work. From this generalization we also propose new algorithms taking into account different criteria for selection of alternatives and different order relations between intervals.

Acknowledgements This work has been partially supported by research grant TIN2010-15055 from the Government of Spain.

\section{References}

1. De Baets, B., Van de Walle, B. , Kerre, E.: Fuzzy preference structures without incomparability. Fuzzy Sets and Systems 16176 (3), 333-348 (1995)

2. Bustince, H., Pagola, M., Barrenechea, E., Fernandez, J., Melo-Pinto, P., Couto, P., Tizhoosh, H.R., Montero, J.: Ignorance functions. An application to the calculation of the threshold in prostate ultrasound images. Fuzzy Sets and Systems 161 (1), 20-36 (2010)

3. Deschrijver, G., Cornelis, C., Kerre, E.E.: On the representation of intuitionistic fuzzy Tnorms and T-conorms. IEEE Transactions on Fuzzy Systems 12(1), 45-61 (2004) 
4. Fodor, J., Roubens, M.: Fuzzy Preference Modelling and Multicriteria Decision Support. Kluwer Academic Publishers (1994)

5. Herrera, F., Martnez, L., Sánchez, P. J.: Managing non-homogeneous information in group decision making. European Journal of Operational Research. 166 (1), 115-132, (2005)

6. Kacprzyk, J.: Group decision making with a fuzzy linguistic majority. Fuzzy Sets and Systems 18, 105-118 (1986)

7. Orlovsky, S. A.: Decision-making with a fuzzy preference relation. Fuzzy Sets and Systems $1(3), 155-167$ (1978)

8. Ovchinnikov, S. V., Roubens, M.: On Strict Preference Relations. Fuzzy Sets and Systems 43, 319-326 (1991)

9. Ovchinnikov, S. V., Ozernoy, , V. M.: Using fuzzy binary relations for identifying noninferior decision alternatives. Fuzzy Sets and Systems 25, 21-32 (1988)

10. Perny, P., Roy, B.: The use of fuzzy outranking relations in preference modelling. Fuzzy Sets and Systems 49, 33-53 (1992)

11. Roubens, M., Vincke, P.: Preference Modelling. In: Lecture Notes in Economics and Mathematical Systems 250. Springer, Berlin (1985)

12. Sambuc, J.: Function $\Phi$-Flous, Application a l'aide au Diagnostic en Pathologie Thyroidienne. These de Doctorat en Medicine, Marseille (1975)

13. Sanz, J., Fernández, A., Bustince, H., Herrera, F.: A genetic tuning to improve the performance of fuzzy rule-based classification systems with interval-valued fuzzy sets: degree of ignorance and lateral position. International Journal of Approximate Reasoning (2011) doi: 10.1016/j.ijar.2011.01.011

14. Szmidt, E., Kacprzyk, J.: Using intuitionistic fuzzy sets in group decision making. Control and Cybernetics 31 1037-1053 (2002)

15. Xu, Z., Yager, R.R.: Some geometric aggregation operators based on intuitionistic fuzzy sets. International Journal of General Systems 35, 417-433 (2006)

16. $\mathrm{Xu}, \mathrm{Z}$.: A method based on distance measure for interval-valued intuitionistic fuzzy group decision making. Information Sciences 180 (1), 181-190 (2010)

17. Zadeh, L. A.: Fuzzy sets. Information Control 8 338-353 (1965)

18. Zadeh, L. A.: The concept of a linguistic variable and its application to approximate reasoning I. Information Sciences 8 199-249 (1975) 